\title{
Liebeskummer tut wirklich weh
}

\author{
Emotionale Tiefschläge wie Liebes- \\ kummer können bei manchen \\ Menschen körperliche Schmerzen \\ verursachen. Wissenschaftler der \\ University of Michigan in Ann Arbor \\ (USA) haben geprüft, ob bei \\ seelischem Kummer die gleichen \\ Hirnareale wie bei körperlichen \\ Schmerzen aktiviert werden.
}

- 40 Probanden der Pilotstudie, die sechs Monate vor den Tests Liebeskummer hatten, bestätigten, dass das daran Denken unangenehm war. Jeder Teilnehmer wurde mit dieser emotionalen Erfahrung erneut konfrontiert. Er nahm außerdem freiwillig an körperlichen Schmerztests teil. Während der Versuche wurde die Gehirnaktivität mit der funktionalen Magnetresonanztomografie (fMRT) gemessen.

Die Probanden sollten Fotos der Person betrachten, die den Liebeskummer ausgelöst hatte. Für die Schmerztests wurden experimentelle Hitzereize am Arm ausgelöst, die schmerzhaft, aber noch erträglich waren.

Wurden die fMRT-Aufnahmen verglichen, dann ähnelten sich die Bilder. Der sog. somatosensorische Kortex für die Schmerzempfindung und die dorsale posteriore Insula für den Liebeskummer sind ähnlich aktiv. In über 500 Aufnahmen wurde zu $88 \%$ eine Übereinstimmung gemessen.

\section{Kommentar}

Die Autoren vermuten, dass auch andere Formen des emotionalen Schmerzes, wie die Trauer um einen Familienangehörigen, ähnliche Aktivitäten auslösen. Der emotionale Schmerz erhält nach diesen Resultaten eine neue funktionelle Dimension.

K. MALBERG "

- E. Kross al.

(Korrespondenz: EE Smith. Department of Psychology, University of Michigan, Ann Arbor, Ml 48109, USA; e-mail: eesmith@psych.columbia. edu) Social rejection shares somatosensory representations with physical pain. Published online before print March 28, 2011 in PNAS; doi:10.1073/pnas.1102693108 PNAS 